\title{
How to answer the question of error margin in forensic signature examination with a Bayesian approach?
}

\author{
RAYMOND MARQUIS \\ School of Criminal Justice \\ Faculty of Law, Criminal Justice and Public Administration \\ University of Lausanne, Switzerland \\ LIV CADOLA \\ School of Criminal Justice \\ Faculty of Law, Criminal Justice and Public Administration \\ University of Lausanne, Switzerland \\ Laboratoire de Recherche en Criminalistique \\ University of Québec at Trois-Rivières, Canada

\section{WILLIAMS DAVID MAZZELLA} \\ School of Criminal Justice \\ Faculty of Law, Criminal Justice and Public Administration \\ University of Lausanne, Switzerland

\section{TACHA HICKS} \\ School of Criminal Justice \\ Faculty of Law, Criminal Justice and Public Administration \\ University of Lausanne, Switzerland

\section{Introduction}

Mandating authorities or parties may ask forensic document examiners to quantify the degree of certainty of their conclusion regarding a signature 
analysis. In recent years, such quantification requests have become more and more prevalent in the current practice of handwriting experts in Switzerland. This highlighted the need to think about a rational answer, and to prevent the usual tendency to avoid the question simply by answering that no statistical numbering are available in the field. This short note reports the approach followed by an examiner to answer such a question, in a case where the Court asked whether a questioned signature was written, or not, by Mr Jones. The Court also required an assessment of the error margin of the signature analysis. What exactly was meant by 'error' was not defined. As this term encompasses a lot of different concepts, we assumed that the Court was interested in the following question: 'What is the probability of being wrong if saying that Mr Jones wrote (or did not write) the questioned signature, after consideration of the examiner's observations?' This question was answered using Bayes' theorem and this case showed that such an approach can be used in court despite the popular belief that Bayes' theorem is beyond what courts may accept.

\section{Comparison findings}

The handwriting examiner was provided with the original questioned document, as well as thirty original reference documents bearing genuine signatures in the name of Mr Jones. The reference documents were business related and covered the period when the purchase agreement was made. The general graphical aspect and the particular features of shape and construction were examined respectively on the questioned signature and on the reference specimens. The reference signatures were homogeneous. The complexity they presented was qualified as medium (Found and Rogers, 1996).

The features of the questioned signature were compared to those observed on the reference specimens, in order to highlight similarities and dissimilarities. Despite the similitude of the general graphical aspect of the signature, many discordant characteristics were found between the questioned signature and the reference specimens, with regards to the particular features of shape and construction of the signature. Furthermore, the questioned signature presented a slightly poorer line quality than the reference specimens, and did not show the pressure variations found on the reference specimens. Many features of the questioned sig- 
nature fell outside the variation range of the reference specimens. Given the case circumstances, disguise was not an issue in this case and thus was not to be considered.

\section{Evaluation}

In this case, in agreement with the guideline of the ENFSI (Willis et al., 2015), the examiner considered the following two propositions to evaluate the comparison findings between the questioned signature and the set of reference signatures: the questioned signature was written by Mr Jones (the proposition of the plaintiff); the questioned signature was simulated by an unknown writer (the proposition of the defendant).

To assign a value to the numerator of the likelihood ratio, i.e., to quantify one's degree of belief of the observations if it is true that the questioned signature was written by Mr Jones, it can be helpful for our thinking to try and answer the following question: how many genuine signatures of Mr Jones should we collect to find one that presents the same kind of findings as the questioned signature? Here, we had observed 30 reference signatures. In the examiner's opinion, it was not expected to find such a series of differences, if the questioned signature was written by Mr Jones, in more than 1 instance among 100 of reference specimens, given that the available reference specimens were homogeneous and adequate for comparison. This value of 0.01 , which accounts for accidental variation, was thus assigned to the numerator of the likelihood ratio.

To assign a value to the denominator of the likelihood ratio, one solution would be to present a set of reference signatures of $\mathrm{Mr}$ Jones to many people and ask them to produce a simulation. The examiner would then determine how many of these simulations show the kind of features observed on the signature under litigation. Because such experimentations are cost and time consuming, they are generally not carried out. Instead, handwriting examiners refer to their training and experience to assess how probable the findings are if the questioned signature was simulated. In the case at hand, it was considered that the observations were expected if the questioned signature was a simulation. The expert's degree of belief of the findings if it was true that the questioned signature 
was indeed a simulation was high. A value of 0.7 was assigned to the denominator of the likelihood ratio.

The value of the likelihood ratio amounts to $1 / 70$. In other words, in the examiner's opinion, the observations are in the order of 70 times more probable if the questioned signature was simulated by an unknown writer, than if it was written by Mr Jones.

\section{Answer to the error margin question}

The opinion of the Court about the propositions, before considering the scientific evidence, are called the prior odds. It is not the role of the expert to assign prior odds, however he proposed some examples to show the impact of the results of his examination on the probability of the propositions. The examiner first considered an example of 50:50 prior odds for the propositions of the plaintiff and the defendant, i.e., the probabilities that Mr Jones signed — or did not sign — the questioned document, before the signature examination. According to Bayes' theorem, these prior odds were multiplied by the likelihood ratio $(\mathrm{LR}=1 / 70)$ assigned by the examiner to the observations made on the documents, to provide posterior probabilities of the propositions, i.e., after considering the signature evidence. By doing so, the posterior probability that Mr Jones did not write the questioned signature would be $98.6 \%$. Conversely, the posterior probability that Mr Jones wrote the questioned signature would be $1.4 \%$. This last value was reported as the probability of being wrong if the Court took the decision that Mr Jones did not write the questioned signature, assuming that the Court's prior odds assignment was 50:50.

As prior odds of 50:50 may not represent the prior odds of the Court, the expert provided two other examples. The second example considered prior odds of 9:1. In this case, the posterior probability that Mr Jones did not write the questioned signature would be $88.6 \%$, and the posterior probability that he wrote it would be $11.4 \%$. In the last example, the expert proposed prior odds of 1:9. In this case, the posterior probability that $\mathrm{Mr}$ Jones did not write the questioned signature would be $99.8 \%$, and the posterior probability that he wrote it would be $0.2 \%$. The probability of being wrong if one takes the decision to say that the questioned signature was simulated by an unknown writer becomes $11.4 \%$ with prior odds of $9: 1$, 
or $0.2 \%$ with prior odds of $1: 9$. One can see that the probability of being wrong, or the error risk in the present case, crucially depends on the Court's prior odds

\section{Conclusion}

It was, in this case, necessary to use numbers to combine the prior odds of the Court to the value of the results given by the expert, help answer to the question of error margin, which was required in percent. Numbers are the only way to handle the complex problem of combining different opinions, in a logical way. Numbers also allow for better consistency and transparency in understanding of expert's results. Indeed, it is now well known that verbal equivalents (taken from verbal scales) are very often misunderstood. A same verbal equivalent, as provided by an expert to convey the value of the evidence, may be perceived differently according to the context and transparency the people (Martire et al. 2013; Martire and Watkins, 2015). That is why we recommend conveying the value of the evidence with numbers in written statements. We propose that a verbal equivalent may be used in addition to the numerical value of the likelihood ratio, and that this verbal equivalent should be taken from a verbal scale that is unified within the institution. These recommendations are fully described in the recent paper of Marquis et al. (2016). We also think that once experts and lawyers will be familiar to the likelihood ratio value as a measure of the weight of evidence (as people are familiar with meters to measure distances), verbal equivalents will no more be useful to convey the value of evidence.

This practical example showed that a logical approach for evidence evaluation can be followed even in a forensic discipline where no numerical data, such as feature frequencies in given populations, are available. This case was also an opportunity to communicate to the fact finder how forensic observations can logically be combined with other elements of the case. 


\section{References}

Found B., Rogers D., "The forensic investigation of signature complexity", [in:] Simner M.L., Leedham C.G., Thomasen A.J.W.M., Handwriting and Drawing Research: Basic and Applied Issues, IOS Press, Amsterdam 1996, pp. 483-492.

Marquis R., Biedermann A., Cadola L., Champod C., Gueissaz L., Massonnet G., Mazzella W.D., Taroni F., Hicks T., "Discussion on how to implement a verbal scale in a forensic laboratory: Benefits, pitfalls and suggestions to avoid misunderstandings", Science and Justice 56 (2016), pp. 364-370.

Martire K.A., Kemp R.I., Watkins I., Sayle M.A., Newell B.R., "The expression and interpretation of uncertain forensic science evidence: Verbal equivalence, evidence strength, and the weak evidence effect", Law and Human Behavior 37 (2013), pp. 197-207.

Martire K.A., Watkins I., "Perception problems of the verbal scale: A re-analysis and application of a membership function approach", Science and Justice 55 (2015), pp. 264-273.

Willis S., Mc Kenna L., Mc Dermott S., O'Donnell G., Barrett A., Rasmusson B., Höglund T., Nordgaard A., Berger C., Sjerps M., Molina J.J.L., Zadora G., Aitken C., Lovelock T., Lunt L., Champod C., Biedermann A., Hicks T., Taroni F., "ENFSI Guideline for evaluative reporting in forensic science", www.enfsi.eu/news/enfsi-guideline-evaluative-reporting-forensic-science (2015).

\section{Summary}

In 2011, a new Criminal Procedure Code was adopted in Switzerland. Since then, forensic handwriting experts more frequently face challenging questions from lawyers. These additional questions especially focus on the degree of certainty regarding the conclusions of the expert, and on the error margin of the signature analysis. The authors seek to address such issues in a scientific manner, in agreement with laws of probability. On the basis of a case, they present the likelihood ratio approach they have followed to evaluate their results, and a full Bayesian approach designed to explain the question of error. The questions of interest were solved with this framework and the use of numbers in a case specific way. The proposed answer aims to explain to the Court what is the probability of being wrong in case at hand. The approach followed is robust, logic and transparent and represents a practical way of addressing the error margin question.

Keywords: questioned documents, signature, interpretation, likelihood ratio, evaluation, Bayes' theorem, error margin. 